\title{
Análisis de legibilidad de formatos de consentimiento informado para pruebas genéticas en México
}

\author{
Beatriz E. de la Fuente-Cortez y Catalina García-Vielma* \\ ${ }^{1}$ Universidad Autónoma de Nuevo León, Facultad de Medicina, Departamento de Genética, ${ }^{2}$ Instituto Mexicano del Seguro Social, Centro de \\ Investigación Biomédica del Noreste, Departamento de Citogenética. Nuevo Léon, México
}

\section{Resumen}

Introducción: Es importante conocer si el documento que ampara el consentimiento informado (Cl) del paciente a quien se le realizan pruebas genéticas de laboratorio (PGL) es legible y comprensible. Objetivo: Analizar la legibilidad de documentos de Cl para pruebas genéticas de laboratorio (PGL) en México. Métodos: Se analizó la legibilidad de 10 formatos de Cl libres en internet utilizados para PGL mediante el programa Legible.es; se evaluó índice de Flesh, versión de Fernández Huerta, y la escala INFLESZ. Se contabilizó el número de sílabas, palabras, frases, párrafos y palabras raras, tiempo para leer el documento y años de escolaridad mínima para entenderlo. Resultados: Se identificó que $60 \%$ de los formatos de $\mathrm{Cl}$ analizados son poco legibles. En promedio, se contabilizaron 3290 sílabas, 1459 palabras, 124 frases, 58 párrafos y 52 palabras raras. El tiempo requerido para la lectura fue de siete minutos y la escolaridad mínima de seis años. Conclusiones: Los formatos de $\mathrm{Cl}$ analizados tuvieron bajos índices de legibilidad y exceden el número recomendado de palabras. Proponemos un modelo de Cl para PGL en México, que cumple con los índices de legibilidad para la correcta comprensión del documento.

PALABRAS CLAVE: Consentimiento informado. Pruebas genéticas. Legibilidad. México

\section{Readability analysis of informed consent forms for genetic tests in Mexico}

\begin{abstract}
Introduction: Knowing if the document that supports the informed consent (IC) granted by the patient who undergoes genetic laboratory tests is legible and understandable is important. Objective: To analyze the readability of IC documents for laboratory genetic tests (LGT) in Mexico. Methods: Readability of 10 free IC forms on the internet used for LGT was analyzed using the Legible.es program; the Flesh index, Fernández-Huerta version, and the INFLESZ scale were evaluated. The number of syllables, words, phrases, paragraphs and strange words, time to read the document and minimum years of education required to understand it were counted. Results: $60 \%$ of the analyzed IC documents were found to have poor readability. On average, 3,290 syllables, 1,459 words, 124 sentences, 58 paragraphs and 52 strange words were counted. The time required for reading it was seven minutes and minimum level of education to understand it was six years. Conclusions: The analyzed IC forms for LGT have low readability rates and exceed the recommended number of words. We propose an IC model for LGT in Mexico that complies with appropriate readability indexes for a correct understanding of the document.
\end{abstract}

KEY WORDS: Informed consent. Genetic tests. Readability. Mexico.

Correspondencia:

*Catalina García-Vielma

E-mail: katygarcia2@ hotmail.com

0016-3813/@ 2020 Academia Nacional de Medicina de México, A.C. Publicado por Permanyer. Este es un artículo open access bajo la licencia CC BY-NC-ND (http://creativecommons.org/licenses/by-nc-nd/4.0/).
Fecha de recepción: 25-02-2020

Fecha de aceptación: 07-07-2020
Gac Med Mex. 2021;157:55-59

Disponible en PubMed

www.gacetamedicademexico.com 


\section{Introducción}

El consentimiento informado $(\mathrm{Cl})$ es un documento en el que el paciente expresa su intención voluntaria, autónoma y responsable de realizarse un procedimiento, como una prueba genética de laboratorio (PGL). ${ }^{1,2}$ Es una vía de comunicación entre el paciente y el médico en el que se expresan compromisos y derechos recíprocos. ${ }^{3}$ Los profesionales de la salud tienen la obligación ética de proporcionar información clara del procedimiento, así como de los posibles riesgos y beneficios, alternativas de tratamiento, consejo genético, etcétera, para que el paciente, a través del $\mathrm{Cl}$, ejerza su derecho y otorgue su permiso, respetando en todo momento su voluntariedad. ${ }^{4,5}$

En México se han dado grandes pasos para establecer que el $\mathrm{Cl}$ sea un documento importante en cuestiones de salud. ${ }^{6}$ En la legislación mexicana, en términos de derecho civil, el $\mathrm{Cl}$ se vincula con los contratos, por lo tanto, obliga a las partes a cumplir con los acuerdos expresamente pactados. ${ }^{7}$ A nivel federal está vinculado con el derecho a la salud, considerado en el artículo 4 de la Constitución Mexicana. ${ }^{8,9}$ En la Norma oficial mexicana NOM-004-SSA3-2012 del expediente clínico se especifican los casos en los que se debe solicitar $\mathrm{Cl}^{10}$ En materia administrativa, está a cargo de la Comisión Nacional de Arbitraje Médico, órgano desconcentrado de la Secretaría de Salud. ${ }^{11,12}$ La protección de datos personales (cuya ley fue publicada en el Diario Oficial de la Federación el 5 de julio de 2010) se considera tanto para el paciente como para sus familiares. ${ }^{13}$

En los últimos años, en el mundo se han implementado diversas PGL en las cuales se manipula información genética. Estas pruebas deben contar con $\mathrm{Cl}$, por lo que es de gran importancia saber si el documento es legible y comprensible para los pacientes. ${ }^{14}$ Por un lado, el abordaje de temas genéticos no siempre es sencillo, ya que su comprensión depende del nivel de escolaridad y factores sociales y culturales, entre otros. Los padecimientos genéticos constituyen un asunto sensible para la familia debido a que algunos no tienen buen pronóstico o las opciones terapéuticas son costosas o experimentales. Por otra parte, la mayoría de los $\mathrm{Cl}$ utilizan un lenguaje técnico, con términos médicos o de laboratorio que no todos los pacientes comprenden, emplean palabras en otro idioma, están escritos con faltas de ortografía o son demasiado extensos e implican demasiado tiempo para que los pacientes los puedan leer y asimilar. ${ }^{14,15}$

Para saber si un escrito es comprensible, hay que conocer su legibilidad lingüística formal, para lo cual se analiza el texto conforme el número de sílabas, palabras, frases, párrafos, etcétera. Existen diversos índices para evaluar la lectura y comprensión de los textos, basados en fórmulas matemáticas que indican que entre más cortas sean las palabras y frases, el texto será más fácil de comprender. ${ }^{15-17}$ Anteriormente, la medición de legibilidad de un texto se realizaba de forma manual; en la actualidad existen programas libres en internet que analizan diferentes índices que nos ayudan con estas mediciones. ${ }^{18-20}$ En la investigación que se presenta, se analizó la legibilidad de algunos $\mathrm{Cl}$ libres en internet, provenientes de empresas y servicios de salud que realizan PGL y brindan servicios en México; además, se propone un modelo de $\mathrm{Cl}$ que cumple con los datos necesarios para la realización de PGL en nuestro país, así como con los requerimientos de legibilidad.

\section{Método}

Se realizó un muestreo intencional de $10 \mathrm{Cl}$ redactados en español, de acceso libre en internet y provenientes de servicios de salud o empresas que realizan PGL y que brindan servicio en México; en la web existen diversos $\mathrm{Cl}$, pero pocos se aplican para la realización de PGL. Para el análisis de legibilidad, cada $\mathrm{Cl}$ fue convertido a archivo de texto en formato Word. El documento fue seleccionado en su totalidad e ingresado en el analizador Legible.es, script de Python de acceso libre en internet, amparado por la General Public License $3 .{ }^{18}$ Este programa realiza un análisis de legibilidad, en el que se evalúa si el texto es fácil de leer y comprender conforme a diversos índices. Además, contabiliza el número de sílabas, palabras, frases, párrafos y palabras raras presentes en el documento, tomando como palabra rara cualquiera mal escrita, con mala ortografía, tecnicismos o en otro idioma diferente al español. También determina el tiempo que el lector invierte para leer el texto y el grado de escolaridad que el lector debe tener para comprenderlo. En el presente trabajo evaluamos el índice de Flesh (versión de Fernández Huerta para documentos en español), ${ }^{19}$ la escala de INFLESZ (índice de Flesch-Szigriszt), la cuenta de sílabas, palabras, frases, párrafos, palabras raras, el tiempo para leer el documento y la escolaridad requerida. ${ }^{20}$ 
Tabla 1. Resultados del análisis de legibilidad por Legible.es

\begin{tabular}{|c|c|c|c|c|c|c|c|c|c|c|c|}
\hline $\begin{array}{l}\mathrm{Cl} \\
\text { (número) }\end{array}$ & Fern & lández Huerta & & FLESZ & Años* & Tiempo't & Sílabas & Palabras & Frases & Párrafos & Palabras \\
\hline 1 & 50.74 & Bastante difícil & 45.84 & Algo difícil & 6.1 & 3.1 & 1409 & 623 & 30 & 39 & 19 \\
\hline 2 & 60.99 & Normal & 56.04 & Normal & 5.7 & 13.0 & 5840 & 2596 & 243 & 119 & 73 \\
\hline 3 & 60.60 & Normal & 55.87 & Normal & 5.9 & 8.9 & 3894 & 1786 & 117 & 60 & 24 \\
\hline 4 & 60.65 & Normal & 55.14 & Normal & 5.0 & 9.0 & 4171 & 1794 & 261 & 115 & 92 \\
\hline 5 & 59.19 & Bastante difícil & 54.37 & Algo difícil & 5.8 & 2.8 & 1287 & 563 & 55 & 21 & 19 \\
\hline 6 & 61.66 & Normal & 56.39 & Normal & 5.5 & 7.2 & 3274 & 1446 & 153 & 78 & 180 \\
\hline 7 & 57.51 & Bastante difícil & 53.03 & Algo difícil & 6.1 & 6.0 & 3863 & 1746 & 108 & 60 & 353 \\
\hline 8 & 57.51 & Bastante difícil & 53.03 & Algo difícil & 6.1 & 6.0 & 2640 & 1197 & 72 & 27 & 23 \\
\hline 9 & 51.9 & Bastante difícil & 47.24 & Algo difícil & 6.6 & 6.1 & 2791 & 1226 & 68 & 15 & 14 \\
\hline 10 & 54.75 & Bastante difícil & 50.1 & Algo difícil & 6.4 & 9.5 & 4307 & 1901 & 121 & 51 & 24 \\
\hline $11^{\star \star}$ & 67.22 & Normal & 62.52 & Normal & 4.6 & 1.8 & 771 & 351 & 46 & 12 & 1 \\
\hline
\end{tabular}

El índice de Fernández-Huerta mide la lecturabilidad de los textos, que significa la legibilidad lingüística del texto, es decir, si es fácil o difícil de entender. Los valores van de 0 a 100 (de muy difícil a muy fácil), relacionándolos de la siguiente forma con el nivel educativo 0 años de estudio: 0-30, muy difícil/universitario; 30-50, difícil/preuniversitario; 50-60, bastante difícil/13-16 años; 60-70, normal/10-12 años; 70-80, bastante fácil/nueve años; 80-90, fácil/seis años; 90-100, muy fácil/cinco años.

La fórmula matemática es la siguiente: ${ }^{19}$

$$
L=206.84-0.60 P-1.02 F
$$

Donde:

$\mathrm{L}=$ lecturabilidad.

$\mathrm{P}=$ promedio de sílabas por palabra.

$\mathrm{F}=$ promedio de palabras por frase.

La escala de INFLESZ mide la facilidad para leer un texto; fue desarrollada y validada por Barrio Cantalejo et al. y está adaptada al lector medio actual. Ha sido utilizada para evaluar otros $\mathrm{Cl}^{16,17}$ Los valores van de 0 a 100 y de muy fácil a muy difícil, relacionándolos con diferentes tipos de publicaciones: 0-40, muy difícil/ universitario-científico; 40-55, algo difícil/bachillerato-divulgación; 55-65, normal/prensa general-deportiva; 65-80, bastante fácil/textos de primaria-novelas; 80-100, muy fácil/educación primaria-cómics. La fórmula es la siguiente: ${ }^{20}$

$$
I=206.835-62.35 S / P-P / F
$$

Donde:

I = escala INFLESZ.

$\mathrm{S}=$ total de sílabas.

$\mathrm{P}=$ cantidad de palabras.

$\mathrm{F}=$ número de frases.

Se realizó estadística descriptiva, calculando medias y desviación estándar de los datos cualitativos y se hizo una comparación de estos.

\section{Resultados}

Los $\mathrm{Cl}$ fueron numerados del 1 al 10 y el resumen de los resultados obtenidos por Legible.es se muestran en la Tabla 1.

De los 10 documentos analizados, seis (60\%) resultaron ser "bastante difíciles" según el índice de Fernández Huerta, mismos que rebasaron la escala INFLESZ, al ser considerados "algo difíciles". Los cuatro $\mathrm{Cl}$ restantes $(40 \%)$ resultaron "normales" en ambos índices de legibilidad.

Los años de estudio necesarios para comprender el documento resultaron en un rango de cinco a 6.6 años $(5.92 \pm 0.43)$ y el tiempo necesario para leerlo fue de 2.8 a 13 minutos $(7.16 \pm 2.92)$.

El número de sílabas en los $\mathrm{Cl}$ analizados varió de 1287 a 5840 (3347.60 \pm 1311.29$)$; el número de palabras tuvo un rango de 563 a 2596 (1487.80 \pm 583.62$)$, el número de frases varió de 30 
a $261(122.80 \pm 73.15)$ y el número de párrafos de 15 a $119(58.50 \pm 34.59)$. El número de palabras raras presentes en el documento fue de 14 a 353 (82.1 \pm 102.95).

Algunos de los $\mathrm{Cl}$ cumplieron con el criterio de legibilidad de Fernández Huerta y la escala INFLESZ, pero empleaban demasiadas palabras y su lectura y comprensión requerían bastante tiempo, como sucedió con el $\mathrm{Cl}$ 2, de una compañía farmacéutica, el cual cumple con los criterios de los índices de legibilidad, pero contiene 5840 sílabas y 2596 palabras y, por lo mismo, se necesitan 13 minutos para leerlo en su totalidad. Por su parte, el Cl 7 contenía 353 palabras raras, entre palabras mal escritas, términos de laboratorio y palabras en inglés.

Nosotros proponemos un formato de $\mathrm{Cl}$ para $\mathrm{PGL}$ en México, con todos los elementos necesarios para que el paciente pueda entender la prueba que se le solicita, respetando en todo momento su voluntariedad, con la respectiva declaración de protección de los datos personales. Este $\mathrm{Cl}$ también fue analizado y resultó con un índice de Fernández Huerta de 67.22 y una puntuación de 62.5 en la escala INFLESZ, lo que lo indica que es un texto "normal" respecto a su legibilidad. El tiempo de escolaridad para entender este documento es de 4.6 años y el tiempo de lectura es de 1.8 minutos, inferior al tiempo de lectura de los $\mathrm{Cl}$ analizados. El número de sílabas, palabras, frases y párrafos es de $771,351,46$ y 12 , respectivamente, y se encontró solo una palabra considerada como rara: "resguardarán", la cual ya fue sustituida por "serán resguardados". Al ser de nuevo analizado, el $\mathrm{Cl}$ propuesto resultó sin ninguna palabra rara.

\section{Discusión}

El legibilidad es una medida indicativa no exacta que ayuda a conocer la calidad del texto que se analiza. Es importante elaborar documentos de $\mathrm{Cl}$ que cuenten con buen nivel de legibilidad, para que los pacientes de todos los niveles educativos y con diferentes niveles sociales y culturales puedan entender el tipo de PGL que se realizará, sus riesgos y beneficios.

De los documentos que analizamos, seis (60\%) no cumplían con los índices analizados y, por lo tanto, eran parcialmente comprensibles 0 difíciles de leer y solo cuatro $(40 \%)$ resultaron con buen nivel de legibilidad. Nuestros resultados son semejantes a los obtenidos en 2013 por Ramírez Puerta et al., quienes analizaron la legibilidad del $\mathrm{Cl}$ de la Unidad de Cuidados Intensivos del Hospital Clínico y Servicio Andaluz de
Salud, en España. Encontraron que, de $28 \mathrm{Cl}$ analizados, solo cuatro (14.2\%) cumplían con todos los índices de legibilidad estudiados (el de Fernández Huerta y el INFLESZ, entre otros). ${ }^{16}$ En 2003, Barrio Canalejo et al. identificaron que cuatro de cada 10 folletos de salud estudiados no cumplían con alguno de los criterios de legibilidad lingüística formal, especialmente el índice de Flesh, debido a que empleaban frases y palabras demasiado largas. ${ }^{15}$

En cuanto al número de palabras, sílabas, frases y párrafos, en 2004 Sharp et al. recomendaron que un formulario de $\mathrm{Cl}$ no debería tener más de 1000 palabras. ${ }^{21}$ En el presente trabajo, en siete de los $10 \mathrm{Cl}$ analizados $(70 \%)$ se excedió el número de palabras recomendado. Los documentos con mayor número de palabras resultan extensos y pueden ser tediosos para el paciente y sus familiares. En 2013, Grazinoli Garrido proporcionó el formato de $\mathrm{Cl}$ a sujetos sometidos a exámenes de ADN y posteriormente aplicó un cuestionario para comprobar la comprensión correcta del documento; $45 \%$ de los sujetos mencionó que no lo había leído. ${ }^{22}$

Trabajos previos indican que existe un bajo índice de legibilidad en los diversos tipos de documentos de información médica. ${ }^{14-17,22}$ La legibilidad se puede mejorar con las siguientes recomendaciones: evitar párrafos largos, usar frases cortas y sencillas, no utilizar abreviaturas ni tecnicismos, evitar la subordinación, la voz pasiva y la doble negación, suprimir el uso de números, símbolos y porcentajes, emplear la mínima cantidad de puntuaciones diferentes al punto y coma, descartar incisos y ofrecer ejemplos prácticos que ilustren conceptos complejos. ${ }^{16,23,24}$

La legibilidad de un documento también se puede afectar por el tipo de letra o su tamaño, entre otros elementos. ${ }^{25}$ Barrio Canalejo et al. encontraron que seis de cada 10 documentos analizados estaban redactados con letra pequeña. ${ }^{15}$ Otros factores que influyen en la comprensión de un documento son el nivel de escolaridad, el nivel sociocultural y los conocimientos en cuestiones de salud, sin dejar de lado los aspectos emocionales del paciente y de su entorno familiar.

Es importante que, como profesionales de la salud, expliquemos con palabras sencillas lo que queremos decir a los pacientes. Si ellos no pueden comprender los documentos que leen, aun cuando se les proporcione la información correcta, su capacidad de decisión de firmar o no el Cl podría verse afectada.

Existen más de 40 sistemas para analizar la legibilidad de los textos, pero hasta donde conocemos, ninguno es especial para textos médicos; sin embargo, 
los sistemas adaptados al español proporcionan una idea de la legibilidad de la información presente en el Cl. ${ }^{16}$

Hasta donde sabemos, no existe ningún otro trabajo que haya realizado análisis de legibilidad en $\mathrm{Cl}$ para PGL en México. Esperamos sentar un precedente al respecto en nuestro país. Asimismo, proponemos un modelo de $\mathrm{Cl}$ que puede servir de base a otros $\mathrm{Cl}$ para diferentes PGL (anexo). Como perspectiva de este trabajo se plantea validar el $\mathrm{Cl}$ propuesto, con la aplicación de un cuestionario a los pacientes para establecer el nivel de comprensión del documento.

\section{Conflicto de intereses}

Ninguno.

\section{Financiamiento}

Ninguno.

\section{Responsabilidades éticas}

Protección de personas y animales. Los autores declaran que para esta investigación no realizaron experimentos en seres humanos ni en animales.

Confidencialidad de los datos. Los autores declaran que siguieron los protocolos de su centro de trabajo sobre la publicación de datos de pacientes.

Derecho a la privacidad y consentimiento informado. Los autores declaran que en este artículo no aparecen datos de pacientes.

\section{Bibliografía}

1. Comisión Nacional de Bioética [Internet]. México: Consentimiento informado. Secretaría de Salud; 2015.
2. López-de la Peña X. El médico y la ley: el consentimiento informado en la legislación en salud de México. Gac Med Mex. 1996;132:553-557.

3. Martínez-Bullé G, Víctor M, coordinadores. Consentimiento informado. Fundamentos y problemas de su aplicación práctica. México: Universidad Nacional Autónoma de México/Instituto de Investigaciones Jurídicas: 2017.

4. Vera-Carrasco OV. El consentimiento Informado del paciente en la actividad asistencial médica. Rev Med La Paz. 2016;22:59-68.

5. Vergés C. Genética y bioética en América Latina. Acta Bioethica. 2004;10:155-166

6. Alonso-Que HT, Aja-Canales J, Castillo-Uribe L, Rodríguez-Delgado NA. El consentimiento informado en la actualidad, su evolución y el punto de vista del experto jurídico. Anales Radiol Mex. 2015;14:172-177.

7. Comisión Nacional de Arbitraje Médico [Internet]. México: Carta de consentimiento válidamente informado; 2019.

8. Acevedo M. Regulación en México del consentimiento informado II. La legislación federal y la Legislación Estatal. México: Universidad Nacional Autónoma de México/Instituto de Investigaciones Jurídicas; 2017.

9. Gobierno de México [Internet]. México: Estatutos mexicanos, libro 4; 2019.

10. Norma Oficial Mexicana NOM-004-SSA3-2012, del expediente clínico. México: Diario Oficial de la Federación 2012 Jun 29.

11. Código Sanitario de los Estados Unidos Mexicanos [Internet]. México: Gobierno de México; 2019.

12. Secretaría de Salud [Internet]. México: Ley General de Salud de México; 2019.

13. Ley General de Protección de Datos Personales en Posesión de Sujetos Obligados. México: Diario Oficial de la Federación; 2017 Ene 26.

14. Blanco-Pérez A, Gutiérrez-Couto U. Legibilidad de las páginas web sobre salud dirigidas a pacientes y lectores de la población en general. Rev Esp Salud Publica. 2002;76:321-331.

15. Barrio-Cantalejo IM y Simón-Lorda P. Medición de la legibilidad de textos escritos: correlación entre método manual de Flesh y métodos informáticos. Aten Primaria. 2003:31:104-108.

16. Ramírez-Puerta MR, Fernández-Fernández R, Frías-Pareja JC, Yuste-Ossorio ME, Narbona-Galdó S, Peñas-Maldonado L. Análisis de legibilidad de consentimientos informados en cuidados intensivos. Medicina intensiva. 2013:37:503-9.

17. Ribeiro-Alves A, Ferrería-Cabrera A. Estudio de Corpus: estructura y legibilidad en el documento de consentimiento informado en el ámbito académico-profesional de las ciencias biomédicas. RLA. 2018;56: $91-116$.

18. Legible.es [Internet]. España: Legible. Analizador de legibilidad de texto; c2019

19. Fernández-Huerta, J. Medida sencilla de lecturabilidad. Consigna. 1959;214:29-32

20. Legibilidad.com [Internet]. Blog analizador de legibilidad de textos; 2016-2018.

21. Sharp SM. Consent documents for oncology trials: does anybody read these things? Am J Clin Oncol. 2004;276:570-575.

22. Grazinoli-Garrido R, Garrido F. Consentimiento informado en genética forense. Acta Bioethica. 2013;19:299-306.

23. Ferrando-Belart V. La legibilidad: un factor fundamental para conocer un texto. Aten Primaria. 2004;34:143-146.

24. Barrio IM, Simón P, Melguizo M, Molina A. Consenso sobre los criterios de legalidad de los folletos de educación para la salud. Anales Sis Navarra. 2011;34:153-165.

25. Paasche MK, Taylor HA, Brancati FL. Readability standards for informed-consent forms as compared with actual readability. N Engl J Med. 2003;348:721-726 


\section{Propuesta de consentimiento informado para realizar pruebas genéticas en México}

\section{Solicitamos su consentimiento para:}

1. Tomar una muestra biológica de (sangre, tejidos o fluidos biológicos) para realizar pruebas genéticas de laboratorio con la finalidad de (diagnosticar, detección de portadores, prueba de paternidad, etcétera)

2. Dichas pruebas serán realizadas en (departamento, servicio u hospital que realice las pruebas).

3. Sus datos estarán protegidos por la Ley de Protección de Datos personales (publicada en el Diario Oficial de la Federación el 26 de enero de 2017). A la muestra se le asignará un número consecutivo, al cual, solo el personal autorizado podrá acceder, así como a los resultados de las pruebas.

4. El médico solicitante, le brindará toda la información necesaria acerca de la toma de muestra, los riesgos de la toma y de la(s) prueba(s) que se realizarán y resolverá todas sus dudas.

5. La información obtenida de estos estudios puede ser importante también para sus familiares, así que, si usted y su familia lo desean, pueden acudir a una consulta especializada en genética, donde se les explicarán los resultados de las pruebas y se les proporcionará asesoramiento genético en caso de ser necesario.

6. Al terminar los análisis, los resultados serán resguardados en el mismo laboratorio que realizó las pruebas. Las muestras excedentes (en caso de que existan), solo si usted lo autoriza, serán almacenadas para futuras pruebas de usted y su familia. En este caso, le presentaremos otro consentimiento informado para saber si otorga la autorización para almacenarlas o para utilizarlas con fines de investigación biomédica. (Si el destino de las muestras sobrantes es su destrucción, debe modificarse este punto).

Si usted comprende la información que se le ha proporcionado, se han resuelto todas sus dudas y otorga su consentimiento para realizar las pruebas genéticas en los términos antes explicados, por favor firme a continuación este consentimiento informado:

Yo: (paciente/padre o madre del paciente/custodio legal del paciente (nombre y dos apellidos)

Acepto que a: (nombre de la persona a quien se le toma la muestra)

Se le tome muestra biológica antes mencionada y doy consentimiento para realizar dichas pruebas genéticas en el Servicio de (departamento, servicio u hospital que realice las pruebas).

Lugar: (lugar de la República Mexicana donde se firma en consentimiento) Fecha: (día) de (mes) de (año).

Nombre y firma del paciente, padre, madre o custodio legal Identificación:
Nombre y firma del, profesional autorizado que solicita el consentimiento.

Identificación:

El presente documento ha sido revisado y aprobado por el Comité de Ética (indicar nombre completo)

Testigo 1

Testigo 2

Nombre y firma

Nombre y firma

0016-3813/@ 2021 Academia Nacional de Medicina de México, A.C. Publicado por Permanyer. Este es un artículo open access bajo la licencia CC BY-NC-ND (http://creativecommons.org/licenses/by-nc-nd/4.0/). 